\title{
Relationship between the real contact behavior and tribological characteristics of cotton fabric
}

\author{
Rongxin CHEN ${ }^{1}$, Jiaxin YE ${ }^{1}$, Wei ZHANG ${ }^{2}$, Jiang WEI ${ }^{1}$, Yan ZHANG ${ }^{1}$, Kun LIU ${ }^{1, *}$ \\ ${ }^{1}$ Institute of Tribology, Hefei University of Technology, Hefei 230009, China \\ ${ }^{2}$ School of Mechanical \& Automotive Engineering, Fujian University of Technology, Fuzhou 350118, China \\ Received: 08 November 2019 / Revised: 06 March 2020 / Accepted: 20 April 2020 \\ (C) The author(s) 2020 .
}

\begin{abstract}
The tribological characteristics of cotton fibers play an important role in engineering and materials science, and real contact behavior is a significant aspect in the friction behavior of cotton fibers. In this study, the tribological characteristics of cotton fibers and their relationship with the real contact behavior are investigated through reciprocating linear tribotesting and real contact analysis. Results show that the friction coefficient decreases with a general increase in load or velocity, and the load and velocity exhibit a co-influence on the friction coefficient. The dynamic change in the real contact area is recorded clearly during the experiments and corresponds to the fluctuations observed in the friction coefficient. Moreover, the friction coefficient is positively correlated with the real contact area based on a quantitative analysis of the evolution of friction behavior and the real contact area at different loads and velocities. This correlation is evident at low velocities and medium load.
\end{abstract}

Keywords: cotton fabric; real contact behavior; tribological characteristics; loading condition; velocity condition

\section{Introduction}

Cotton fibers are the purest sources of cellulose, comprising more than $90 \%$ cellulose [1]. Compared with other fibers, cotton fibers are more widely used in engineering, materials science, and biology, particularly in applications such as composite fiber bearings and carbon fabric composite functional materials [2-4]. Cotton fibers are classified as viscoelastic materials, and exhibit complex mechanical behaviors. The tribological characteristics of cotton fibers are fundamental to their mechanical behavior, and are crucial for understanding their surface characteristics. The friction behavior reflects the ability of fibers to withstand shear motion, and is influenced by the structure of the fiber surface, loading type, and relative motion velocity [5]. Furthermore, understanding the macrofriction behavior of cotton fibers is an essential step in the realization of superior material properties, such as wear resistance, tear resistance, and lifespan [6]. Hence, understanding the tribological characteristics of cotton fibers is necessary to improve fiber processing technology and the performance of fiber products.

The properties of cotton fibers have been investigated in previous studies, but its tribological properties are unknown and require exploration [7-9]. Several studies have explored the friction mechanisms of fibers. The friction between the skin of a finger and fibers has been investigated using tactile feedback and a macrotribometer [10-13]. Zhang et al. [14] investigated the tribological interactions between surgical suture and artificial skin and found that the structure and surface topography of

* Corresponding author: Kun LIU, E-mail: liukun@hfut.edu.cn 
surgical suture can determine the tribological properties. The friction coefficient and friction force have been analyzed in accordance with the different types of fibers, fingers, and skins. However, these studies have focused on face-to-face contact and the influence of the skin material. In addition, these studies have not been concerned with different contact types and the real contact interface of fabrics. The microfriction behaviors of single and multiple fibers have been investigated [15, 16], while the effects of the radius of a single fiber, pulling force, and pulling speed on friction have been analyzed. Such studies have primarily focused on the contact between each fiber and the micromorphology of a single fiber. Observing the real contact interface of a single fiber is difficult, and the microfriction behaviors cannot fully reflect the macrotribological characteristics. Numerous studies [17-21] have been conducted on the tribological properties of fabric composites to reveal the material properties of the composites, including surface wettability, thermal properties, wear performance, and the contents of different substances. Fabric composites exhibit unique friction behavior compared with regular fabrics, as well as satisfactory wear resistance and self-lubricating properties. However, prior studies have not considered the macrotribological characteristics of the original fibers.

Overall, the above studies on fibers have largely ignored the real contact interface and the relationship between the real contact behavior and tribological characteristics. However, the macrofriction behaviors of cotton fabrics require comprehensive investigation. Typically, the surfaces of cotton fabrics are rough because of cross fibers. The real contact behavior of fibers involves the evolution of the contact area between the asperities of two surfaces, which influences the tribological characteristics, including friction force, friction coefficient, and surface properties [7, 22-25]. Hosseinali and Thomasson [7] found that dimensional properties influence the friction coefficient of a single fiber. The friction coefficient has a negative relationship with the micronaire value, and the real contact area is small in high-micronaire fibers. Bueno et al. [25] determined the influence of different fiber cross-section shapes on roughness and reported that a large real contact area is related to the considerable roughness of a round-shaped fiber. Smerdova and Sutcliffe [26] investigated the change in the micro contact area at different fabric shear angles and approximated the contact area using the ideal contact length and half of the contact width. These studies are minimally concerned with the accurate quantitative analysis of the relationship between the real contact area and friction coefficient of cotton fabrics, and pay more attention to the real contact area of a single fiber.

In the present study, reciprocating linear tribotesting was performed to analyze the macrotribological properties of cotton fibers and the real contact behavior between the cotton fibers and a planoconvex glass lens. The influences of load and velocity on the tribological characteristics were investigated, and the macrotribological characteristics of cotton fabrics were obtained. Subsequently, the dynamic evolution of the real contact area was recorded, and its relationship with the friction coefficient was qualitatively explored. Finally, the relationship between the real contact area and friction coefficient was quantitatively analyzed.

\section{Experiments}

\subsection{Material}

The cotton fabrics (weight: $80 \mathrm{~g} / \mathrm{m}^{2}$, yarn count: $40 \mathrm{~s}$, and thread per inch: $80 \times 60$ ) used in this study were made of $100 \%$ cotton and obtained from Guangzhou Xintianji Textile Co., Ltd., China. These materials were pure and used without further treatment. The size of each sample was $5 \mathrm{~cm} \times 3 \mathrm{~cm}$; however, our experimental analyses focused on a $2 \mathrm{~mm} \times 1 \mathrm{~mm}$ region in the center of the sample. Images of a cotton fabric under different magnifications and a scanning electron microscopy (SEM) images of a single fiber are shown in Fig. 1.

\subsection{Tribotesting method}

A reciprocating linear tribometer was used to measure friction; the cotton fabrics were loosely placed on the bottom moving platform to realize the reciprocating motion along the $x$-direction in accordance with the selected velocity. An image of 

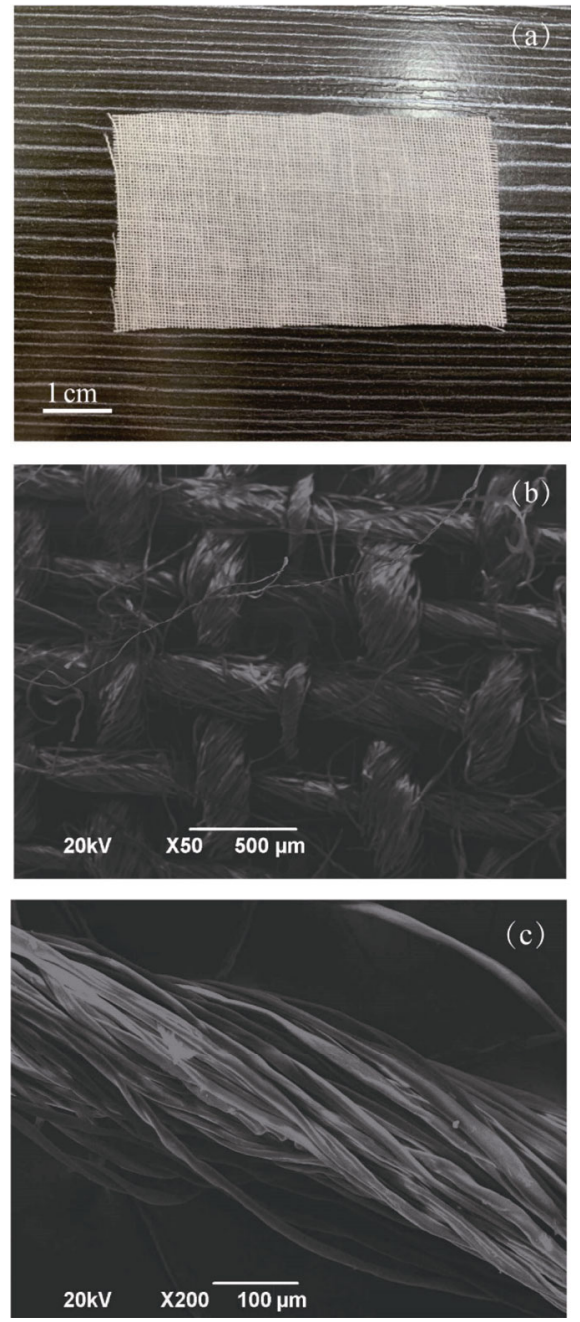

Fig. 1 Images of a cotton fabric under (a) low and (b) high magnification, and (c) SEM image of a single fiber.

the tribotester is shown in Fig. 2(a). The bottom moving platform was controlled using a steppermotor controller (MD14MRQ23A7, Intelligent Motion Systems, Inc., UK). A three-way force sensor (JDSH-2, Bengbu Sensor System Co., Ltd., China) was clamped onto the platform to measure the tangential and normal forces. A $2.5 \mathrm{~mm}$-radius plano-convex glass lens $\left(\mathrm{SiO}_{2}\right.$, curvature radius: $4.8 \mathrm{~mm}$, focal length: $10 \mathrm{~mm}$, Beijing Hengding Light Technology Co., Ltd., China), which was in contact with the cotton fabric, was glued to the underside of the top moving platform. The height of the top moving platform was adjustable along the $z$-direction and used to apply the desired normal load. During the friction tests, the glass lens was fixed at a given height. Moreover, a high-speed camera (MER-131210U3M/C-L, 60 fps, Daheng [Group] Co., Ltd., Beijing Image Visual Technology Branch, China) was used to capture the images of the contact interface between the glass lens and cotton fabric dynamically.

A schematic of the cotton fiber tribology test setup is shown in Fig. 2(b). During the friction tests, a piece of pure cotton fabric was placed on the bottom moving platform, and the position of the cotton fabric was adjusted until it was centered beneath the camera. Subsequently, the cotton fabric was subjected to the desired normal load beneath the lens by adjusting the height of the top moving platform. Because the glass lens was glued to the underside of the top moving platform. The normal force $\left(F_{\mathrm{N}}\right)$ and tangential force $\left(F_{\mathrm{t}}\right)$ were recorded in real time using LabVIEW software. The height of the high-speed camera was adjusted until a clear image of the contact interface appeared. Finally, the different loads, velocities, and the fixed displacement $(2 \mathrm{~mm})$ of the cycles were set (Table 1), and the images of the dynamic contact interface were collected. According to the Amontons-
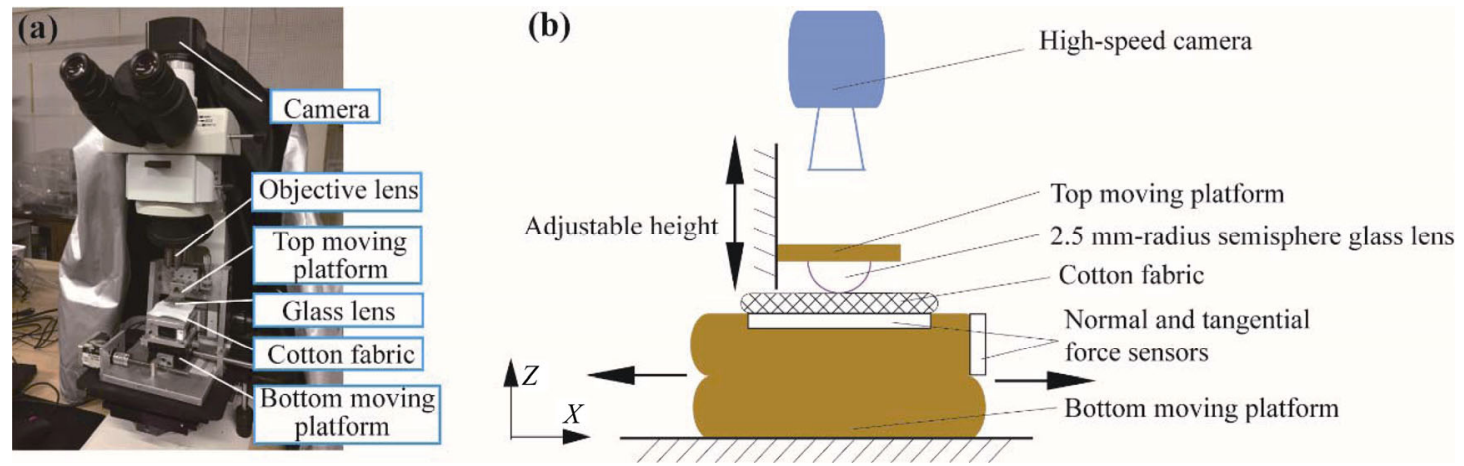

Fig. 2 (a) Image of the tribotester and (b) schematic of the cotton fiber tribology test setup. 
Coulomb linear friction model, the friction coefficient $(\mu)$ was calculated using the ratio of the tangential force to the normal force, as follows:

$$
\mu=\frac{F_{\mathrm{t}}}{F_{\mathrm{N}}}
$$

\subsection{Real contact analysis techniques}

It was necessary to determine the scale of the images before capturing them. A standard-sized metal sample was placed on the bottom moving platform to obtain the pixels of the dimensions of the square area and match them to the corresponding real dimensions. Subsequently, the height of the high-speed camera was adjusted to a suitable position to observe a standard fabric sample and obtain the standard area $\left(S_{1}\right)$ and the pixels of the area $\left(n_{1}\right)$ using a $5 \times$ objective lens. Images of the original contact interface were captured using the highspeed camera and subjected to binarization, as shown in the original image (Fig. 3(a)). The area of the real contact was identified by comparing the differences between pixels, before the RGB value was changed to $(0,0,0)$, as shown in the processed image (Fig. 3(b)). Finally, the pixels of the real contact area $\left(n_{2}\right)$ were obtained, and the real contact area $\left(S_{2}\right)$ was calculated as follows:

Table 1 Details of the experimental parameters.

\begin{tabular}{cc}
\hline Variables & Value \\
\hline Load $(\mathrm{N})$ & $1,2,3,4,5,6,7$ \\
Velocity $(\mathrm{mm} / \mathrm{s})$ & $0.2,0.3,0.4,0.5,0.6,0.7,0.8,0.9,1.0$ \\
Displacement $(\mathrm{mm})$ & 2 \\
Cycles & 20 \\
\hline
\end{tabular}

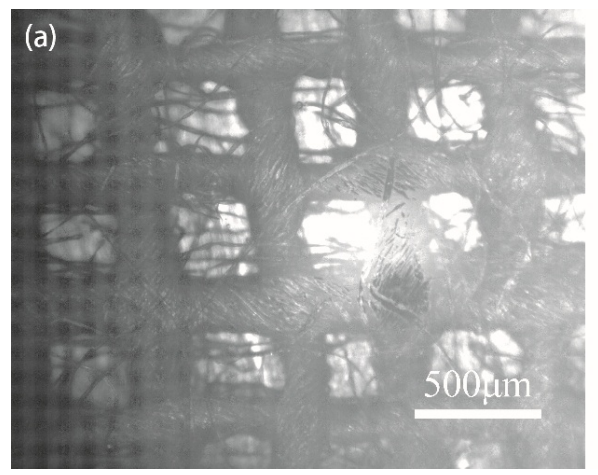

Fig. 3 Images of (a) original and (b) processed contact area.

$$
S_{2}=\frac{n_{2}}{n_{1}} \times S_{1}
$$

\section{Results and discussion}

\subsection{Effects of load and velocity on the tribological characteristics}

Figure 4 shows the influence of load and velocity on the friction coefficients. Under different constant loads, the friction coefficients initially decrease with increasing velocity, then stabilize, before increasing slightly (Fig. 4(a)). The lowest friction coefficients are recorded at a velocity of $0.6 \mathrm{~mm} / \mathrm{s}$. The number of contact points has a significant influence on the friction of the fibers [27]. Xiang et al. [28] showed that the number of contact points affects the friction coefficient of glass-fiber woven fabrics. Hermann et al. [29] discussed the influence of velocity on the friction coefficient of fabrics on the basis of contact points and conditions. Thus, the change in the friction coefficient with velocity may be attributed to a change in the number of contact points. As the velocity increases, the number of contact points gradually decreases, and the contact area decreases, thus accounting for the decrease in friction coefficients with the increase in velocity from 0.2 to $0.6 \mathrm{~mm} / \mathrm{s}$, i.e., relatively low velocities. The glass lens and cotton fibers are in contact across the general contact region. The elastic deformation recovers in the no-contact region, and since the contacts separate completely, the corresponding friction coefficient within this velocity range is relatively low. When the velocity increases from 0.6 to $1 \mathrm{~mm} / \mathrm{s}$, i.e., to relatively high

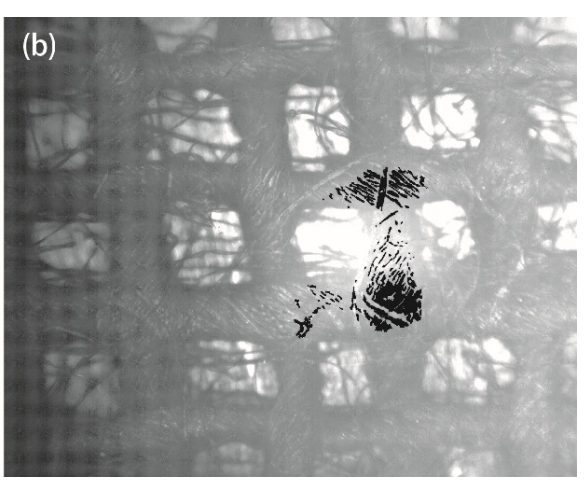



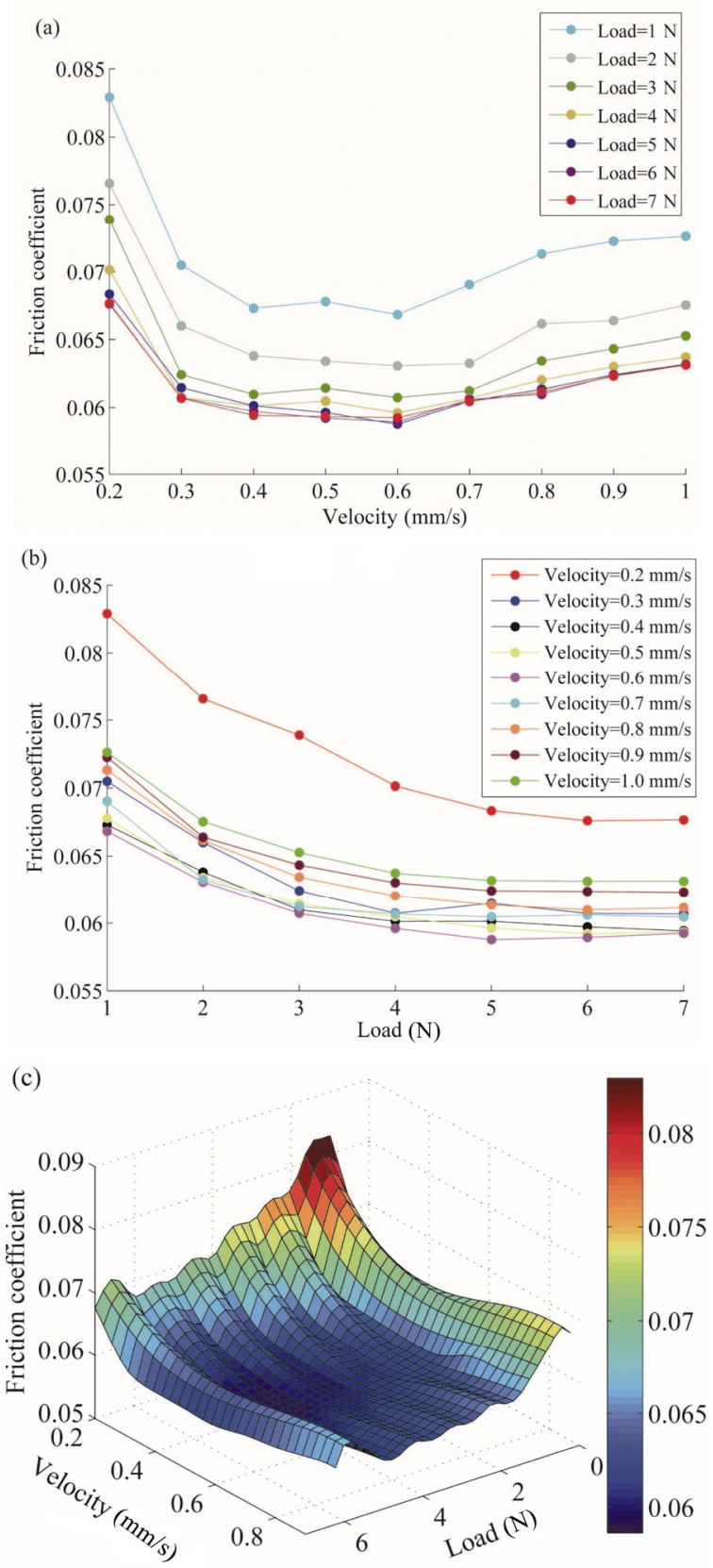

Fig. 4 Effects of (a) load, (b) velocity, and (c) co-influence of load and velocity on the friction coefficients.

velocities, the elastic deformation partly recovers in the no-contact region, and the contacts do not separate completely. The number of all-contact points increases, and the corresponding friction coefficient increases slightly. The number of contact points at different velocities will be the subject of future studies. The evolution of the friction coefficient and corresponding real contact area at different sliding velocities are analyzed and discussed in
Section 3.3. Nishimatsu and Sawaki [30] found that the friction coefficient first decreases and then increases slightly with increased velocity. Their findings are consistent with our results. However, Hermann et al. [29] reported that the sliding speed has no significant effect on the friction coefficient, determined by pulling across the fabric sample using a sled of polymethyl methacrylate (PMMA) surface. They reported a remarkable fluctuation in the sliding speed with this experimental condition, which diminished its effect.

Moreover, the friction coefficients decrease gradually at different constant velocities with increasing load (Fig. 4(b)). As the load increases, the contact area becomes smooth. The contact area of a single contact point increases, but the number of contact points, and accordingly, the friction coefficient, decrease. Ajayi and Elder [31] reported similar decreasing trends in the friction coefficient with increased load for different fabrics. The co-influence of load and velocity on the friction coefficient of the cotton fabric is shown in Fig. 4(c). Low load and velocity result in a high friction coefficient. The load and velocity have nearly similar influence levels.

\subsection{Dynamic evolution of real contact behavior}

The change in the friction coefficient at a velocity of $0.2 \mathrm{~mm} / \mathrm{s}$ and under a load of $3 \mathrm{~N}$ was analyzed based on the tribotesting method to determine the dynamic change in the friction behavior. Figure 5 indicates that the surface morphologies of the cotton fabric before and after testing using Phenom XL-type SEM equipment are similar under the above conditions. This result can be attributed to the limited test cycles. Figure 6(a) shows a graph with the friction coefficient as a function of time, indicating the cyclical nature of the testing process. The evolution of the friction coefficient with time is similar for every cycle. At every cycle, the positive friction coefficient fluctuates and increases with time, following which the absolute value of the negative friction coefficient fluctuates and increases with time. Figure 6(b) shows eight data points of a half cycle at different displacements, illustrating the variable behavior of the friction 

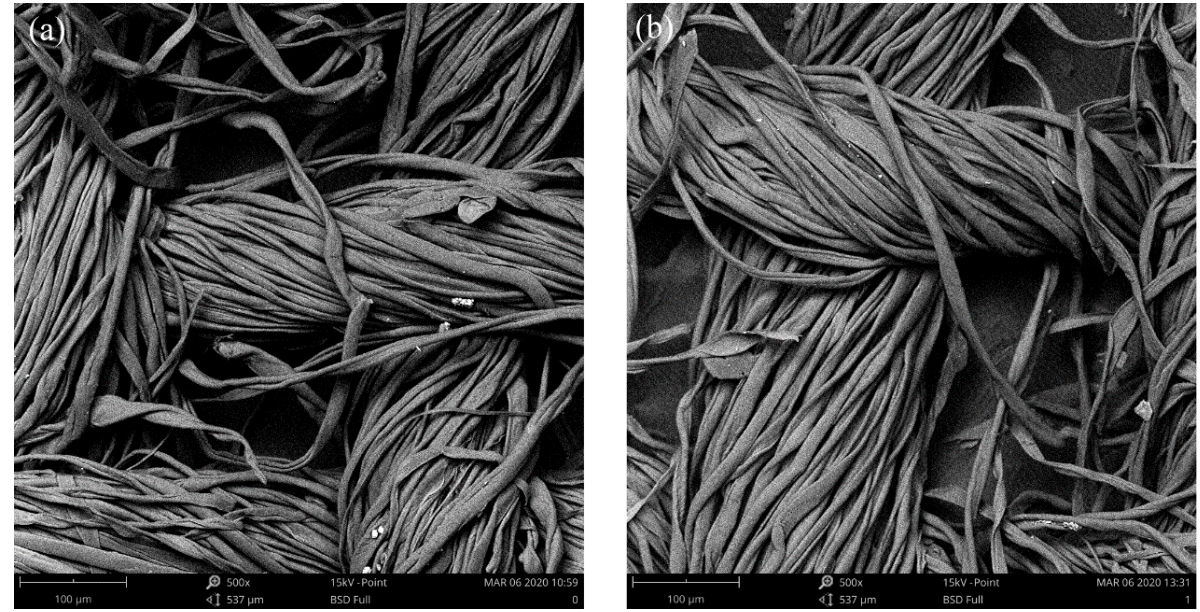

Fig. 5 Surface morphology of cotton fabric (a) before and (b) after tribotesting.
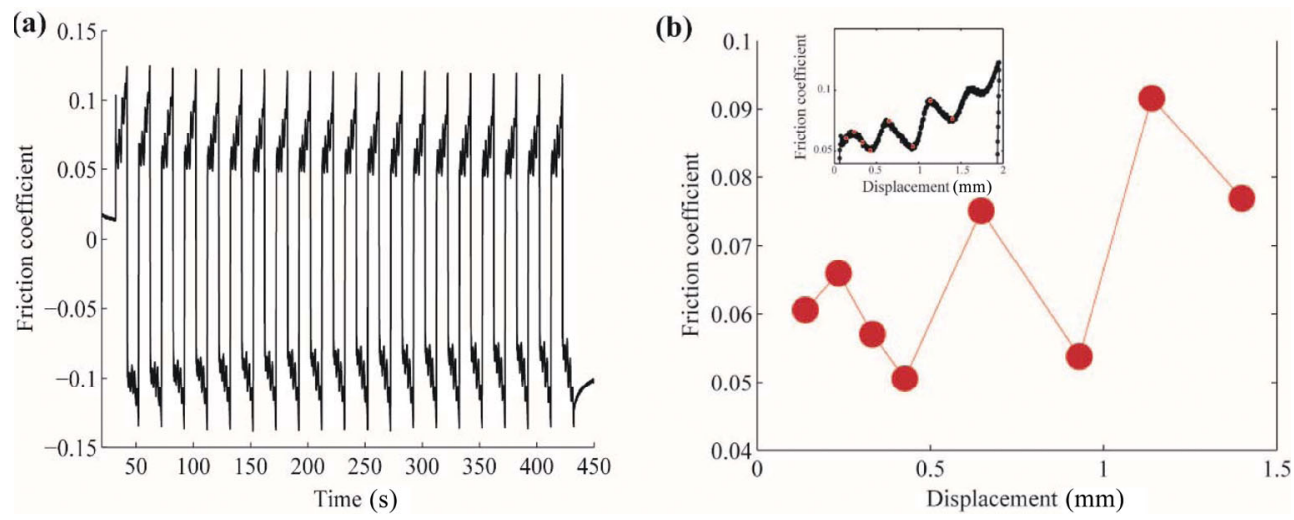

Fig. 6 Change in friction coefficient with (a) time and (b) displacement at a velocity of $0.2 \mathrm{~mm} / \mathrm{s}$ and load of $3 \mathrm{~N}$.

coefficient. The change in the friction coefficient for an entire half cycle is shown in the inset of Fig. 6(b). In order to evaluate the relationship between the real contact area and the friction coefficient accurately, limited data points were selected for analysis. The eight data points that describe the general fluctuating trend of the friction coefficient for a half cycle were chosen accordingly. Owing to the relatively sharp change observed in the friction coefficient at the end of a half cycle, no data points were selected from this stage.

According to the inset of Fig. 6(b), the friction coefficient fluctuates and increases with time throughout the half cycle. On the one hand, the fluctuating behavior of the friction coefficient may be explained by the stick-slip phenomenon displayed by cotton fabric, which is attributed to its viscoelastic properties on a microscale [32]. Thus, the friction coefficient demonstrates a fluctuating tendency similar to stick-slip behavior. On the other hand, the increase in the friction coefficient with time may be ascribed to a typical system error experienced by tribology testers, associated with the dip angle of the tester platform [33]. The small dip angle of the tester platform can, therefore, account for the increase in the friction coefficient with time. The real friction coefficient was recorded to analyze the relationship between the friction coefficient and real contact area.

The dynamic evolution of the real contact area, which corresponds to the displacement in Fig. 6(b), was obtained based on the above real contact analysis techniques (Fig. 7). The insets in Fig. 7 display the local magnification of the respective contact areas. The experimental parameters (velocity and load) used to obtain the images in Fig. 7 were the same as those used for Fig. 6(b). The friction coefficient fluctuates between 0.04 and 0.1 (Fig. 6(b)). From 0.139 to $0.235 \mathrm{~mm}$, the friction coefficient increases, and the corresponding contact area enlarges. 
Moreover, from 0.235 to $0.333 \mathrm{~mm}$, the friction coefficient decreases, and the corresponding reduction in contact area manifests in a synchronized change in trends.

Generally, the friction coefficient increases and decreases, as the contact area enlarges and reduces, respectively. A high friction coefficient corresponds to a large contact area, which is discussed further in the next section.

The cotton fabric is a plain cloth, which is composed of wefts and warps, i.e., the horizontal and vertical threads, respectively. The difference in the contact areas between the weft and warp based on the test results was investigated. The contact area concentrated on the wefts (along the horizontal direction) is small, and that on the warps (along the vertical direction) is large. The relative motion direction between the glass lens and the surface of the cotton fabric is perpendicular to the warp, thereby causing the warp to bunch up, enlarging its relative contact area. In contrast, the relative motion direction is parallel to the weft, causing the weft to loosen, reducing its contact area. The relative motion direction may influence the contact area and is related to the friction coefficient; relevant research will be conducted in the future.

\subsection{Relationship between real contact areas and friction coefficients}

The relationship between the change in the friction
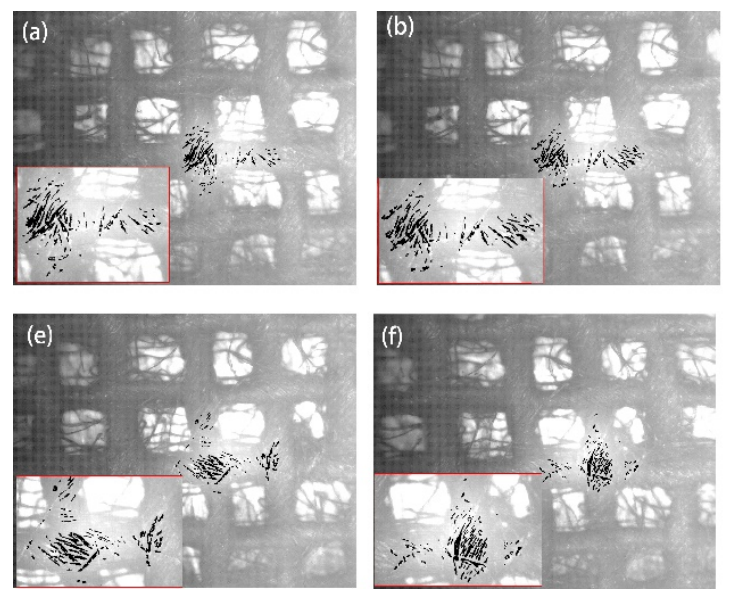

coefficient and real contact area in Section 3.2 is quantitatively investigated in this section to determine the relationship between the contact behavior and friction (Figs. 8 and 9).

The friction coefficient and the contact area demonstrate similar change behavior at a velocity of $0.2 \mathrm{~mm} / \mathrm{s}$ under different loads (Fig. 8). A high friction coefficient is associated with a large contact area. The Pearson correlation coefficients [34] are $0.39,0.84$, and 0.41 under loads of 1,3 , and $7 \mathrm{~N}$, respectively. Therefore, the friction coefficient and contact area are positively correlated, and this relationship is remarkable under a load of $3 \mathrm{~N}$. As evident from the results of Figs. 8(a)-8(c), the entire contact area increases with increasing load. Given that a large load enhances and smoothens the contact interface, the associated friction coefficient is low, consistent with the low friction coefficient associated with large loads (Fig. 4(b)).

The friction coefficient and contact area also demonstrate similar change behavior under a load of $3 \mathrm{~N}$ at different velocities (Fig. 9). A high friction coefficient is associated with a large contact area. The friction coefficient and contact area are positively correlated at different velocities. The Pearson correlation coefficients [34] are $0.84,0.90$, and 0.53 at velocities of $0.2 \mathrm{~mm} / \mathrm{s}, 0.5 \mathrm{~mm} / \mathrm{s}$, and $0.8 \mathrm{~mm} / \mathrm{s}$, respectively. The correspondence is closest at low velocities. According to Fig. 4(a), a large contact area is associated with a high friction coefficient.

In general, the friction coefficient and contact
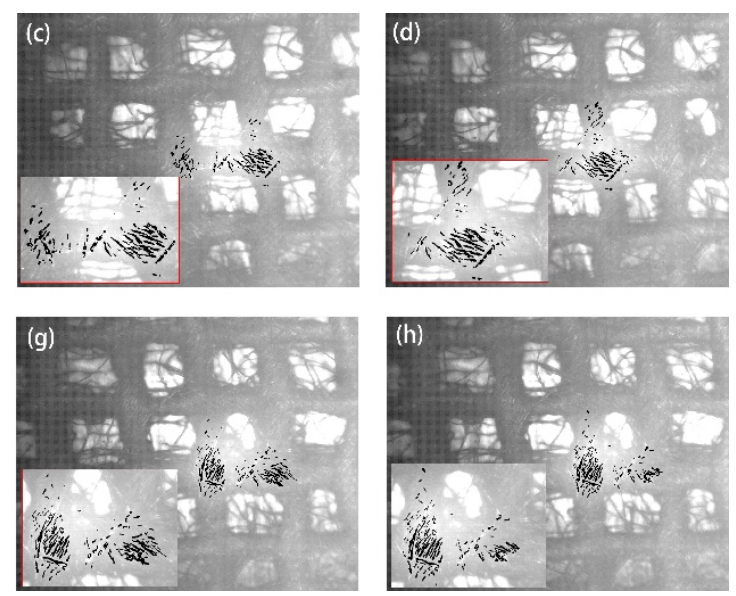

Fig. 7 Dynamic evolution of contact area as a function of displacement $(D)$ : (a) $D=0.139 \mathrm{~mm}$, (b) $D=0.235 \mathrm{~mm}$, (c) $D=$ $0.333 \mathrm{~mm}$, (d) $D=0.426 \mathrm{~mm}$, (e) $D=0.647 \mathrm{~mm}$, (f) $D=0.93 \mathrm{~mm}$, (g) $D=1.139 \mathrm{~mm}$, and (h) $D=1.399 \mathrm{~mm}$. 

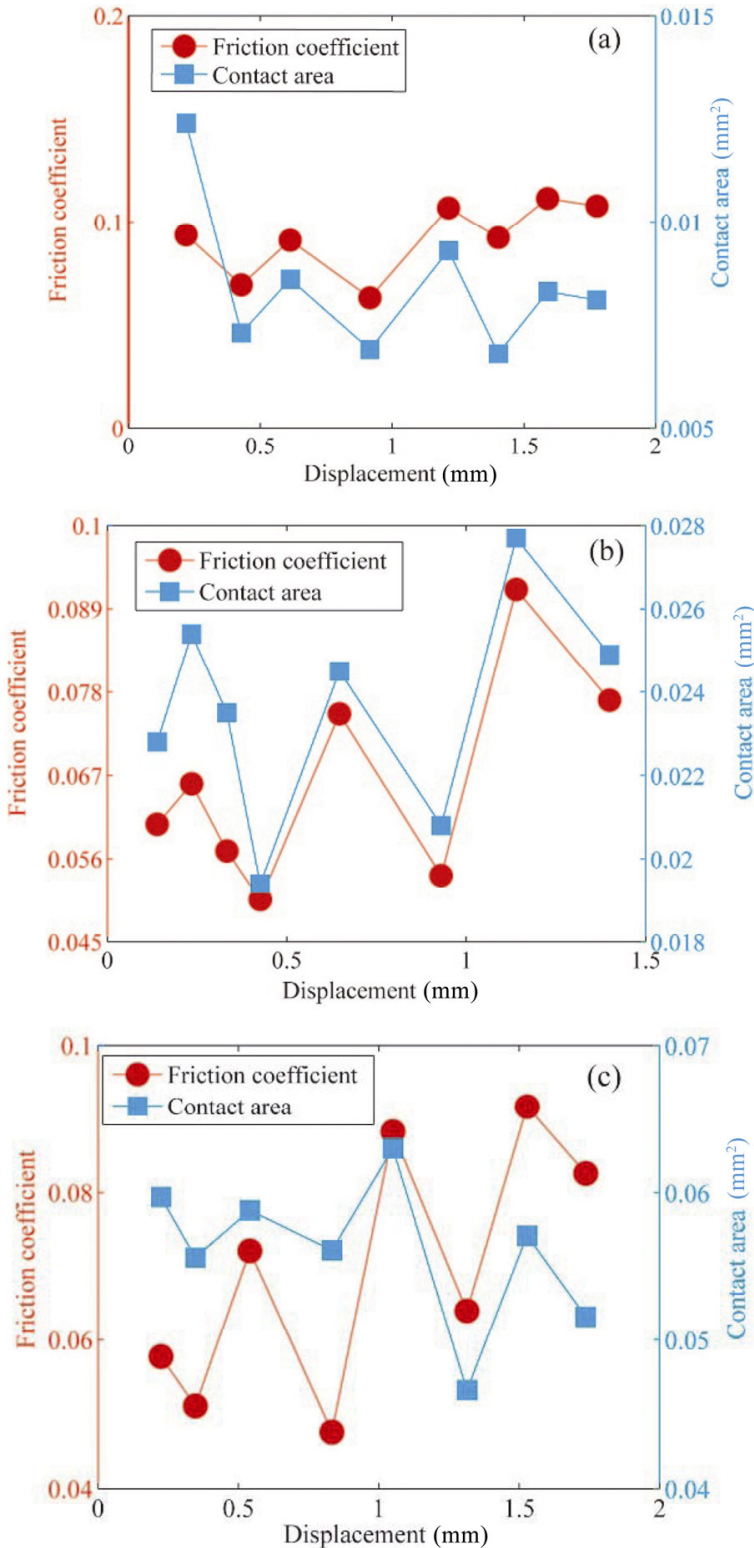

Fig. 8 Correspondence between the friction coefficient and contact area at a velocity of $0.2 \mathrm{~mm} / \mathrm{s}$ and loads of (a) $1 \mathrm{~N}$, (b) $3 \mathrm{~N}$, and (c) $7 \mathrm{~N}$, respectively.

area are positively correlated, which can be attributed to the mechanical characteristics of the cotton fiber and contact mode. The contact density increases with the deformation of fibers when they come in contact with the glass lens, and the corresponding contact area increases. The fibers possess the properties of viscoelastic bodies [35] and the friction force is related to the sum of all real contact areas as per the adhesion theory of friction [7]. The adhesion of fibers is enhanced with increased contact area, thereby increasing the
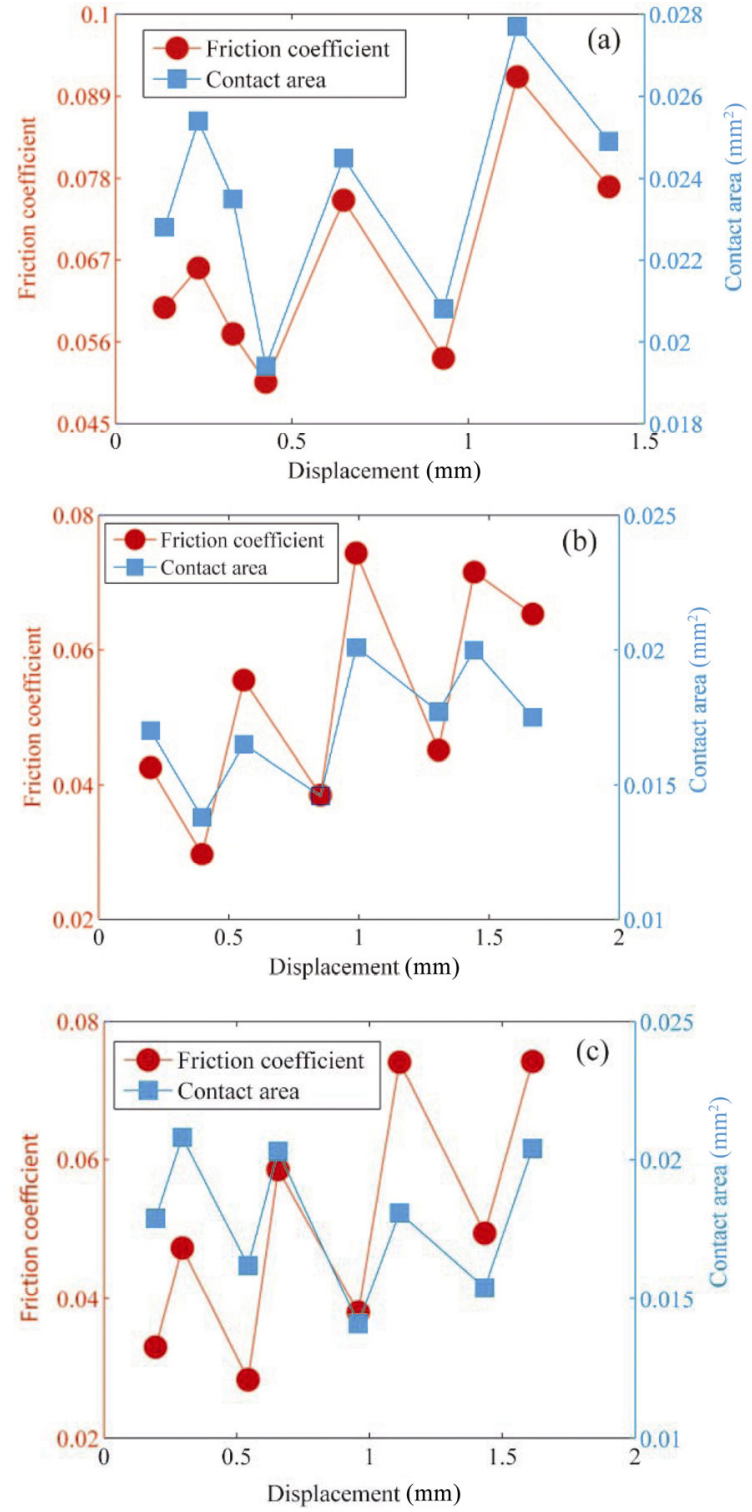

Fig. 9 Correspondence between the friction coefficient and contact area under a load of $3 \mathrm{~N}$ and velocities of (a) 0.2 $\mathrm{mm} / \mathrm{s}$, (b) $0.5 \mathrm{~mm} / \mathrm{s}$, and (c) $0.8 \mathrm{~mm} / \mathrm{s}$, respectively.

friction coefficient. Moreover, the literature shows that the friction coefficient increases with an increase in the contact area. A positive correlation exists between the friction coefficient and the contact area [26, 36], but their quantitative relationship has not been sufficiently investigated.

\section{Conclusions}

The friction behavior between cotton fabric and a plano-convex glass lens was investigated through reciprocating linear tribotesting. The contact interface 
was observed using a high-speed camera and the influence of load and velocity on the friction coefficient was analyzed. The real contact area between friction pairs was determined using real contact analysis techniques. Moreover, the relationship between the friction coefficient and real contact area was established. Based on the results, the following conclusions were drawn:

1) Load and velocity considerably influence the friction coefficient between the cotton fabric and glass lens. The friction coefficient decreases gradually with increasing load. This phenomenon is related to the decrease in the number of contact points. With an increase in velocity, the friction coefficient decreases, before increasing again when the velocity exceeds $0.6 \mathrm{~mm} / \mathrm{s}$. This result may be attributed to the elastic deformation recovery of the cotton fiber.

2) The friction coefficient fluctuates with displacement, and the real contact areas, which are determined using real contact analysis techniques, respond to this changing trend. Moreover, the relative motion direction between the glass lens and the surface of the cotton fabric may influence the real contact area.

3) The contact area is positively correlated with the friction coefficient under different loads or different velocities. This phenomenon is attributed to the mechanical characteristics of the cotton fiber and contact mode. Moreover, the correspondence is remarkable at low velocities and under medium load.

\section{Acknowledgements}

The authors are grateful to the National Natural Science Foundation of China (Nos. 51875152 and 51975174) for financial support of this study.

Open Access: This article is licensed under a Creative Commons Attribution 4.0 International License, which permits use, sharing, adaptation, distribution and reproduction in any medium or format, as long as you give appropriate credit to the original author(s) and the source, provide a link to the Creative Commons licence, and indicate if changes were made.

The images or other third party material in this article are included in the article's Creative Commons licence, unless indicated otherwise in a credit line to the material. If material is not included in the article's Creative Commons licence and your intended use is not permitted by statutory regulation or exceeds the permitted use, you will need to obtain permission directly from the copyright holder.

To view a copy of this licence, visit http:// creativecommons.org/licenses/by/4.0/.

\section{References}

[1] Lewin M. Handbook of Fiber Chemistry. Boca Raton (USA): CRC Press, 2006.

[2] Avila A G, Hinestroza J P. Smart textiles: tough cotton. Nat Nanotechnol 3(8): 458 (2008)

[3] Aminayi P, Abidi N. Imparting super hydro/oleophobic properties to cotton fabric by means of molecular and nanoparticles vapor deposition methods. Appl Surf Sci 287: 223-231 (2013)

[4] Ramalho A, Szekeres P, Fernandes E. Friction and tactile perception of textile fabrics. Tribol Int 63: 29-33 (2013)

[5] Zhang Z Y, Fletcher I W, Hurley C R, Boardman C, Doyle P, Leggett G J. Morphological and quantitative frictional measurements of cotton fibres using friction force microscopy. J Mater Chem 20(39): 8531 (2010)

[6] Latif W, Basit A, Ali Z, Baig S A. The mechanical and comfort properties of cotton and regenerated fibers blended woven fabrics. Int $J$ Cloth Sci Tech 30(1): 112-121 (2018)

[7] Hosseinali F, Thomasson J A. Variability of fiber friction among cotton varieties: Influence of salient fiber physical metrics. Tribol Int 127: 433-445 (2018)

[8] Thomasson J A, Mengüç M P, Shearer S A. Radiative transfer model for relating near-infrared and micronaire measurements of cotton fibers. TASABE 38(2): 367-377 (1995)

[9] Aslan M, Yamada J, Meng-uacute M P. Characterization of individual cotton fibers via light-scattering experiments. J Thermophys Heat Tr 17(4): 442-449 (2003)

[10] Kim M S, Kim I Y, Park Y K, Lee Y Z. The friction measurement between finger skin and material surfaces. Wear 301(1-2): 338-342 (2013)

[11] Darden M A, Schwartz C J. Investigation of skin 
tribology and its effects on the tactile attributes of polymer fabrics. Wear 267(5-8): 1289-1294 (2009)

[12] Camillieri B, Bueno M A. Artificial finger design for investigating the tactile friction of textile surfaces. Tribol Int 109: 274-284 (2017)

[13] Li W L, Shen H Y, Hung J T, Shih C P. The effect of moisture on friction coefficient of fabrics used on taekwondo personal protective equipment. P I Mech Eng J-J Eng 233(1): 87-94 (2019)

[14] Zhang G Q, Ren T H, Zeng X Q, Heide E V D. Influence of surgical suture properties on the tribological interactions with artificial skin by a capstan experiment approach. Friction 5(1): 87-98 (2017)

[15] Viswanathan, A. 5-Frictional forces in cotton and regenerated cellulose fibres. Journal of the Textile Institute Transaction 57(1): T30-T41 (1966)

[16] Ramkumar S S, Umrani A S, Shelly D C, Tock R W. Study of the effect of sliding velocity on the frictional properties of nonwoven fabric substrates. Wear 256(3-4): 221-225 (2004)

[17] Gu D P, Zhang L X, Chen S W, Song K F, Liu S Y. Reciprocating sliding wear of hybrid PTFE/Kevlar fabric composites along different orientations. RSC $A d v$ 8(37): 20877-20883 (2018)

[18] Li D, Guo Z. Robust superhydrophobic and selflubricating PTES-TiO 2@ UHMWPE fabric and its tribological properties. $R S C A d v$ 7(15): 9169-9175 (2017)

[19] Xiong X S, Shen S Z, Alam N, Hua L, Li X, Wan X J, Miao M H. Mechanical and abrasive wear performance of woven flax fabric/polyoxymethylene composites. Wear 414: 9-20 (2018)

[20] Luo Z J, Song B J, Han J Y, Yan S Z. An experimental method for quantitative analysis of real contact area based on the total reflection optical principle. Chinese Phys B 28(5): 054601 (2019)

[21] Wang Z Q, Jing N I, Gao D R. Combined effect of the use of carbon fiber and seawater and the molecular structure on the tribological behavior of polymer materials. Friction 8(2): 396-420 (2020)

[22] Vijay R, Singaravelu D L, Jayaganthan R. Development and characterization of stainless steel fiber-based copper-free brake liner formulation: A positive solution for steel fiber replacement. Friction 6(2):183-194 (2018)

[23] Pei X Q, Lin L Y, Schlarb A K , Bennewitz R. Contact area and shear stress in repeated single-asperity sliding of steel on polymer. Tribol Lett 67(1): 30 (2019)

[24] Li W B, Huang J F, Fei J, Liang Z H, Cao L Y, Yao C Y. Study on tribological properties as a function of operating conditions for carbon fabric wet clutch. Tribol Int 94: 428-436 (2016)

[25] Bueno M A, Aneja A P, Renner M. Influence of the shape of fiber cross section on fabric surface characteristics. J Mater Sci 39(2): 557-564 (2004)

[26] Smerdova O, Sutcliffe M P F. Multiscale tool-fabric contact observation and analysis for composite fabric forming. Compos Part A-Appl S 73: 116-124 (2015)

[27] El Mogahzy Y E, Gupta B S. Friction in fibrous materials: Part II: experimental study of the effects of structural and morphological factors. Text Res $J$ 63(4): 219-230 (1993)

[28] Xiang Z, Liu Y, Zhou X, Wu Z, Hu X. Interlayer contact mechanism of the frictional behavior of glass-fiber woven fabrics and improvements of winding characteristics. Compos Struct 233: 111497 (2020)

[29] Hermann D, Ramkumar S S, Seshaiyer P, Parameswaran S. Frictional study of woven fabrics: The relationship between the friction and velocity of testing. $J$ Appl Polym Sci 92(4): 2420-2424 (2004)

[30] Nishimatsu T, Sawaki T. Study on pile fabrics. Journal of the Textile Machinery Society of Japan 30(1): 13-17 (1984)

[31] Ajayi J O, Elder H M. Fabric friction, handle, and compression. J Text I 88(3): 232-241 (1997)

[32] Hosseini Ravandi S A, Toriumi K, Matsumoto Y. Spectral analysis of the stick-slip motion of dynamic friction in the fabric surface. Text Res $J$ 64(4): 224-229 (1994)

[33] Burris D L, Sawyer W G. Addressing practical challenges of low friction coefficient measurements. Tribol Lett 35(1): 17-23 (2009)

[34] Azéma E, Radjai F. Force chains and contact network topology in sheared packings of elongated particles. Phys Rev E 85(3): 031303 (2012)

[35] Fuller K N G, Tabor D. The effect of surface roughness on the adhesion of elastic solids. Proceedings of the Royal Society A: Mathematical 345(1642): 327-342 (1975)

[36] Smerdova O, Sutcliffe M P F. Novel experimental method for microscale contact analysis in composite fabric forming. Exp Mech 55(8): 1475-1483 (2015) 


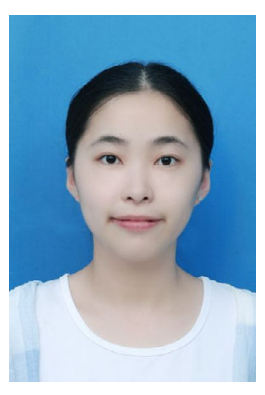

Rongxin CHEN. She received her bachelor degree in aerocraft manufacture engineering in 2017 from Hefei University of Technology,
Hefei, China. Now, she is a Ph.D. student in the Institute of Tribology at the same university. Her research interests include interfacial wetting and imbibition.

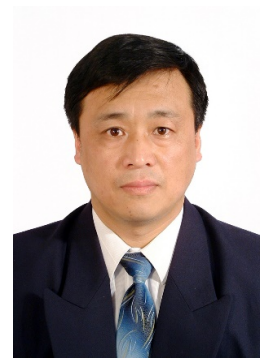

Kun LIU. He received his M.S.

Theory and Bearings in 1995 from Xi'an Jiaotong degree in 1988 from Hefei University, $\mathrm{Xi}^{\prime}$ an, China. His current position is a University of Technology, Hefei, China, and received his Ph.D. professor and the director of the Institute of degree in Institute of Lubrication Tribology. His research area mainly focuses on the tribology design. 